Andrew R. Wolf Bronagh Blackwood Brian Anderson

\section{Tolerance to sedative drugs in PICU: can it be moderated or is it immutable?}

Received: 20 October 2015

Accepted: 21 October 2015

Published online: 24 November 2015

(c) Springer-Verlag Berlin Heidelberg and ESICM 2015

\section{A. R. Wolf ( $)$}

Department of Anaesthesia and Intensive Care, Our Lady's Children's Hospital, Crumlin, Dublin 12, Ireland

e-mail: awolfbch@aol.com

\section{B. Blackwood}

School of Medicine, Dentistry and Biomedical Sciences, Queens University, University Road, Belfast BT7 1NN, Northern Ireland, UK

\section{B. Anderson}

Department of Anaesthesiology, University of Auckland FHMS, Park Road Grafton, Auckland 1023, New Zealand

\section{Dear Editor,}

Nienke Vet and colleagues have evaluated the influence of daily sedation interruptions (DSI) in PICU [1]. This was ambitious: bias is hard to avoid and recruitment rates in such studies are poor, which ultimately restrict the strength of conclusions. Nevertheless, their results suggest that, if behavioural tools already drive sedation delivery, then DSI will not substantially influence the duration of ventilation. These findings are consistent with recent reports from adult intensive care [2].

A pause in drug delivery allows plasma concentration to fall followed by reduction in effect site concentration and subsequent arousal. However, the lag time can be significant (Fig. 1a). Recovery may be further delayed by the presence and influence of midazolam's alpha-hydroxy metabolite that has half the activity of the parent drug [3]. Other drugs used for sedation also have influence: a three- drug combination (e.g. midazolam, propofol, and alfentanil) can triple the duration of effect compared with propofol alone [4]. In the current study, additional sedative and analgesic drugs were used "as required". This may have contributed to the lack of impact from midazolam interruption. Variation in recovery from sedation is complicated by the high prevalence of renal failure, hepatic failure and concomitant administration of CYP3A inhibitors in PICU patients. Sedation requirements vary widely not only with age, diagnosis and clinical state, but also between similar patients.

Sedation has parallels with innovations in postoperative pain control in children: intermittent drug use was replaced by continuous infusions, then patient- and nursecontrolled analgesia (PCA/NCA) with pain score monitoring. Further optimization combined low dose continuous infusions modulated by PCA/NCA. PICU sedation is in need of similar improvement but can this impact on patient outcomes? Practitioners of anaesthesia are also aware of emergence delirium complicating recovery. Could a similar phenomenon complicate midazolam interruption, necessitating reintroduction of the drug prematurely?

The value of DSI [5-7] remains unclear. The results of this current study could simply reflect the strong history in the Netherlands of good sedation practice including a relative sparing use of sedatives. This is supported by the relatively low dose of midazolam used compared to a previous paediatric study [7]. One conclusion is that recovery from sedation relates to the overall cumulative "sedation burden" and that minimizing the exposure, with effective behavioural scoring linked to delivery, optimizes recovery irrespective of DSI and other factors.

Interventions such as those described by Vet and colleagues [1] are complex. They consist of multiple linked elements: [8] context and setting (workload, resources, staffing); ICU staff characteristics (skill mix, training); and clinical processes (complexity of proocols, 
algorithms, decision-making and perceived risk) [9]. Understanding these components is critical to interpreting and generalising study findings [10]. This study does not provide contextual information that may help explain the results. An indication of the degree of compliance and nursing perspectives about the DSI may assist with interpreting the findings. Qualitative studies exploring non-adherence to DSI have highlighted a lack of nursing acceptance due to patient agitation and the subsequent risk of adverse events associated with more wakeful patients [11, 12]. The UK Medical Research Council recommends that a process evaluation should accompany trials of complex interventions [10].

Can tolerance be manipulated by modifying practice? Techniques that should be considered include: avoiding drugs particularly associated with tolerance such as midazolam; moderating early exposure to high doses of analgesics and sedatives to prevent acute tolerance; drugsparing strategies such as drug cycling or polypharmacy mixtures; and returning to low-dose propofol, a drug that has been legislated out of PICU in many countries.
Midazolam remains the most popular sedative despite a rate of association with withdrawal phenomena of up to $35 \%$ [13]. The frequency and severity of tolerance and withdrawal is related to the cumulative "drug burden" and higher doses $(>300 \mu \mathrm{g} / \mathrm{kg} / \mathrm{h})$ [14]. A sedated patient is thought to be easier to nurse [15] and, even in this current study, children were sedated with mean midazolam doses $(183-240 \mu \mathrm{g} / \mathrm{kg} / \mathrm{h})$ to the deeper end of the sedation scale. There are alternatives to midazolam. Alpha-2 agonists can provide effective sedation either as a straight midazolam replacement or as a supporting drug $[16,17]$. Currently, there are no substantial data to determine whether other drug combinations can reduce tolerance and accelerate recovery.

Acute exposure to high doses of high-efficacy shortacting opioids (e.g. fentanyl, remifentanil) is linked to accelerated drug tolerance [18] and delay in recovery. Fast-track paediatric cardiac surgery has moved away from high-dose fentanyl, $100 \mu \mathrm{g} / \mathrm{kg}$ or more, to $10-15 \mu \mathrm{g} /$ $\mathrm{kg}$ over the perioperative period resulting in earlier extubation and accelerated recovery. There are potentially
Fig. 1 a Plasma concentrations and effect in a 2-year-old child given protocol midazolam bolus $(0.1 \mathrm{mg} / \mathrm{kg})$ and infusion changes $(100 \mu \mathrm{g} / \mathrm{kg} / \mathrm{h}$ step changes) every $30 \mathrm{~min}$ to achieve sedation. Infusion was stopped at $180 \mathrm{~min}$. Sedation recovery lags behind the decline in plasma concentration. Amplitudes in the $11.5-30 \mathrm{~Hz}$ (beta) frequency band were used as an EEG effect measure. Pharmacodynamic parameter estimates from Mandema et al. [2]. b Plasma concentrations and effect in a neonate given protocol midazolam bolus $(0.1 \mathrm{mg} / \mathrm{kg})$ on two early occasions (5-min interval) to achieve sedation. Plasma concentration declines slowly because of slow clearance. Sedation recovery lags way behind the decline in plasma concentration even though a maintenance infusion was not even given. Pharmacodynamic parameter estimates were from Mandema et al. [2]
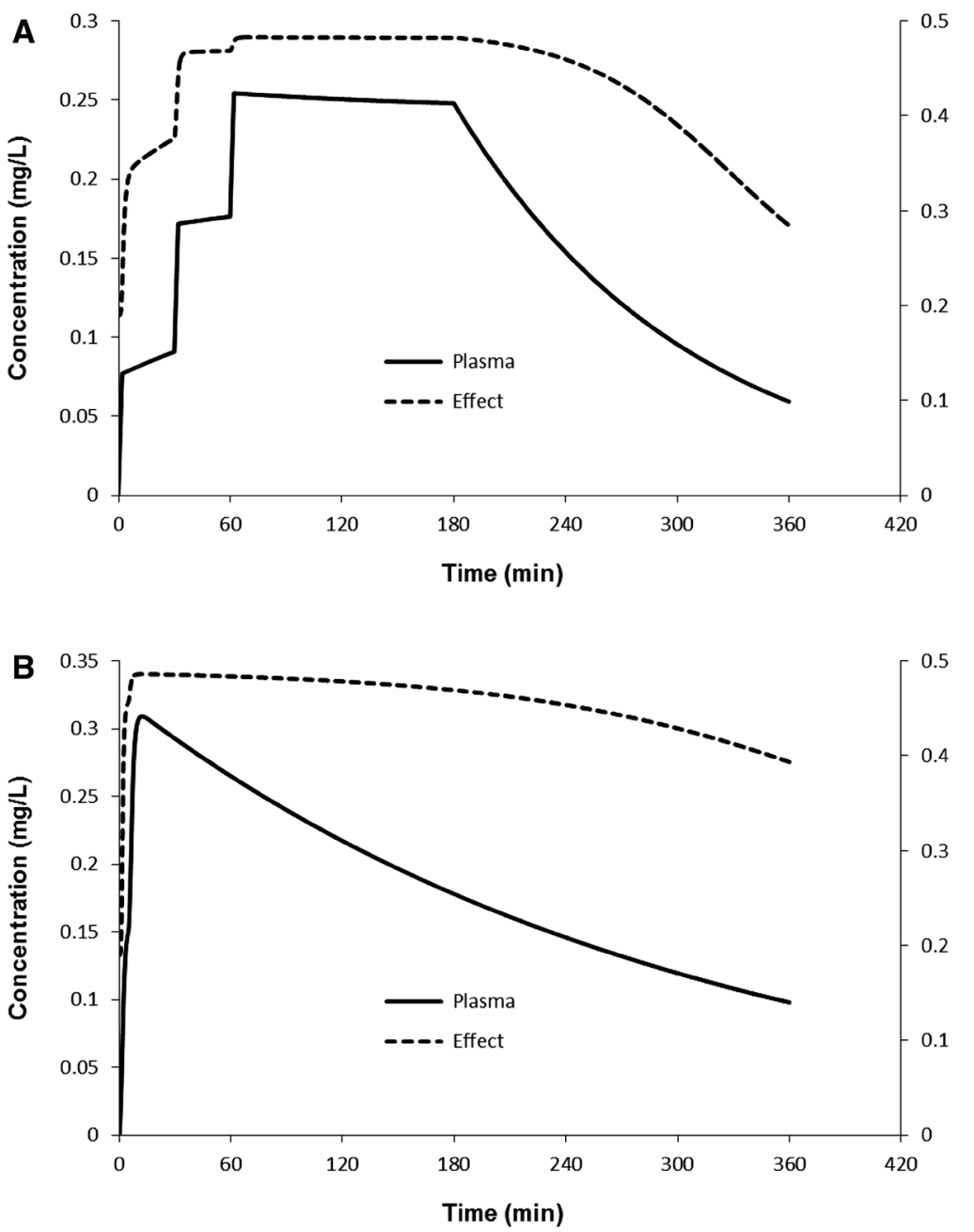
transferable lessons from this experience that could reduce tolerance in PICU patients. Drug delivery must reflect the age- and context-dependent pharmacokinetics. For example, while drug delivery in the young infant needs to be high during the loading phase, downward adjustment is necessary during maintenance, reflecting reduced elimination compared to the older infant (Fig. 1b). Unfortunately, behavioural scoring may be impossible in this early phase if neuromuscular blocking drugs are used, and higher doses than necessary may be continued. Rotating drug sedation and analgesic regimens, or using non-pharmacological strategies to maintain comfort, have been used to try to limit drug requirements. While this approach makes sense, there are no data to support it.

The SLEEPs trial [16] demonstrated that neither morphine/midazolam nor morphine/clonidine alone could always provide complete sedation. A third agent was often required. The choice of the third agent may be important; in the current study, multiple rescue drugs were used. While propofol as a major sedative agent has been eliminated from use in PICU due to fears of propofol infusion syndrome, it continues to be used cautiously by some, even in countries where the drug is officially discouraged. Low-dose infusion $(0-4 \mathrm{mg} / \mathrm{kg} / \mathrm{h})$ with careful surveillance for accumulation and lactic acidosis deserves to be reconsidered as a third-line drug.

Sedation is often treated as a necessary evil in PICU; the primary disease and its treatment is naturally the main focus while sedation is managed generically. During recovery, the secondary problems associated with sedation (e.g. nosocomial infection, poor gut motility and behavioural change) become lost within the disease and general PICU experience. Under-sedation and over-sedation are both harmful, while minimising sedation exposure and their adverse effects are important. Optimised matching of delivery to sedation requirement provides another marginal gain in the critically ill child that can contribute to improving patient outcomes.

\section{Compliance with ethical standards}

Conflicts of interest On behalf of all authors, the corresponding author states that there is no conflict of interest.

\section{References}

1. Vet Nienke J, de Wildt Saskia N, Verlaat Carin WM et al (2015) A randomized controlled trial of daily sedation interruption in critically ill children. Intensive Care Med. doi: 10.1007/s00134-015-4136-z

2. Burry L, Rose L, McCullagh IJ et al (2014) Daily sedation interruption versus no daily sedation interruption for critically ill adult patients requiring invasive mechanical ventilation. Cochrane Database Syst Rev 7:CD009176

3. Mandema JW, Tuk B, van Steveninck AL et al (1992) Pharmacokineticpharmacodynamic modeling of the central nervous system effects of midazolam and its main metabolite alpha-hydroxymidazolam in healthy volunteers. Clin Pharm Ther 51:715-728

4. Minto CF, Schnider TW, Short TG et al (2000) Response surface model for anesthetic drug interactions. Anesthesiology 92:1603-1616

5. Mehta S, Burry L, Cook D (2012) Daily sedation interruption in mechanically ventilated critically ill patients cared for with a sedation protocol: a randomized controlled trial. JAMA 308:1985-1992
6. Verlaad CW, Heesen GP, Vet NJ et al (2014) Randomised controlled trial of daily interruption of sedatives in critically ill children. Pediatr Anesth 24:151-156

7. Gupta K, Gupta VK, Jayashree $M$ et al (2012) Randomised controlled trial of interrupted versus sedative infusions in ventilated children. Pediatr Crit Care Med 13:131-135

8. Blackwood B (2006) Methodological issues in evaluating complex health care interventions. J Adv Nurs 54(5):612-622

9. Rose L, Blackwood B, Burns SM, Frazier SK, Egerod I (2011) International perspectives on the influence of structure and process of weaning from mechanical ventilation. Am J Crit Care 20(1):e10-e18. doi: 10.4037/ajcc2011xxx

10. Moore GF, Audrey S, Barker M, Bond L, Bonell C, Hardeman W, Moore L, O'Cathain A, Tinati T, Wight D, Baird J (2015) Process evaluation of complex interventions: medical research council guidance. BMJ 350:h1258. doi: 10.1136/bmj.h1258
11. Everingham K, Fawcett T, Walsh T (2014) Targeting' sedation: the lived experience of the intensive care nurse. J Clin Nurs 23:694-703

12. Tanios M, de Wit M, Epstein SK, Devlin JW (2009) Perceived barriers to the use of sedation protocols and daily sedation interruption: a multidisciplinary survey. J Crit Care 24:66-73

13. Hughes J, Gill A, Leach HJ et al (1994) A prospective study of the adverse effects of midazolam on withdrawal in critically ill children. Acta Paediatr 83(11):1194-1199

14. Jenkins IA, Playfor SD, Bevan $C$ et al (2007) Current United Kingdom sedation practice in pediatric intensive care. Paediatr Anaesth 17(7):675-683

15. Sneyers B, Laterre PF, Bricq E et al (2014) What stops us from following sedation recommendations in intensive care units? A multicentric qualitative study. J Crit Care 29:291-297 
16. Wolf A, McKay A, Spowart C et al (2014) Prospective multi-centre randomised, double-blind, equivalence study comparing clonidine and midazolam as intravenous sedative agents in critically ill children: the SLEEPS Study (Safety ProfiLe, Efficacy and Equivalence in Paediatric intensive care Sedation). Health Technol Assess 18(71):1-212
17. Achuff BJ, Nicolson SC, Elci OU, Zuppa AF (2015) Intraoperative dexmedetomidine reduces postoperative mechanical ventilation in infants after open heart surgery. Pediatr Crit Care Med 16(5):440-447
18. Crawford MW, Hickey C, Zaarour C, Howard A, Naser B (2006) Development of Acute opioid tolerance during remifentanil infusion for pediatric scoliosis surgery. Anesth Analg 102(6):1662-1667 